BMJ Open Sport \& Exercise Medicine

\title{
DEPA classification: a proposal for standardising PRP use and a retrospective application of available devices
}

\author{
J Magalon, ${ }^{1,2}$ A L Chateau, ${ }^{1,2}$ B Bertrand, ${ }^{3}$ M L Louis, ${ }^{4}$ A Silvestre,${ }^{5}$ L Giraudo, ${ }^{1}$ \\ J Veran, ${ }^{1}$ F Sabatier ${ }^{1,2}$
}

To cite: Magalon J, Chateau AL, Bertrand B, et al. DEPA classification: a proposal for standardising PRP use and a retrospective application of available devices. BMJ Open Sport Exerc Med 2016;2:e000060. doi:10.1136/bmjsem-2015000060

- Prepublication history for this paper is available online. To view these files please visit the journal online (http://dx.doi.org/10.1136/ bmjsem-2015-000060).

Accepted 1 January 2016

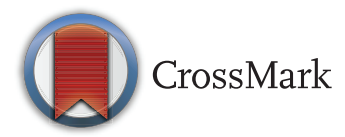

${ }^{1}$ Cell Culture and Therapy Laboratory, Hôpital de la Conception, AP-HM, CIC BT 1409, Marseille, France ${ }^{2}$ Vascular Research Center of Marseille, Aix-Marseille University, Marseille, France ${ }^{3}$ Plastic Surgery Department, Hôpital de la Conception, APHM, Marseille, France

${ }^{4}$ ICOS, Sport and Orthopedics Surgery Institute, Marseille, France ${ }^{5}$ Radiology Department, Bordeaux Merignac Sports Clinic, Merignac, France

Correspondence to Dr Jérémy Magalon; jeremy.magalon@ap-hm.fr

\section{ABSTRACT}

Background/aim: Significant biological differences in platelet-rich plasma (PRP) preparations have been highlighted and could explain the large variability in the clinical benefit of PRP reported in the literature. The scientific community now recommends the use of classification for PRP injection; however, these classifications are focused on platelet and leucocyte concentrations. This presents the disadvantages of (1) not taking into account the final volume of the preparation; (2) omitting the presence of red blood cells in PRP and (3) not assessing the efficiency of production.

Methods: On the basis of standards classically used in the Cell Therapy field, we propose the DEPA (Dose of injected platelets, Efficiency of production, Purity of the PRP, Activation of the PRP) classification to extend the characterisation of the injected PRP preparation. We retrospectively applied this classification on 20 PRP preparations for which biological characteristics were available in the literature.

Results: Dose of injected platelets varies from 0.21 to 5.43 billion, corresponding to a 25 -fold increase. Only a Magellan device was able to obtain an A score for this parameter. Assessments of the efficiency of production reveal that no device is able to recover more than $90 \%$ of platelets from the blood. Purity of the preparation reveals that a majority of the preparations are contaminated by red blood cells as only three devices reach an A score for this parameter, corresponding to a percentage of platelets compared with red blood cells and leucocytes over $90 \%$.

Conclusions: These findings should provide significant help to clinicians in selecting a system that meets their specific needs for a given indication.

\section{INTRODUCTION}

The potential role of platelet-rich plasma (PRP) in enhancing the healing of bone, muscle, ligaments and tendons, has resulted in multiple applications within virtually all the orthopaedic subspecialties. Several uncontrolled studies have shown benefit for

\section{What are the new findings?}

- Dose of injected platelets varies from 0.21 to 5.43 billion, depending on the device used.

- Efficiency of the platelet-rich plasma (PRP) preparation does not reach $90 \%$ of platelet recovery no matter which device is used.

- Some available devices furnish more red blood cells than platelets in their PRP.

a variety of indications ${ }^{1}{ }^{2}$ and more recently controlled studies have demonstrated lessfavourable results. ${ }^{3} 4$ A common point between these studies is the lack of biological characterisation of the content of the PRP used as therapy product.

Marx,${ }^{5}$ first described PRP as a suspension of platelets in plasma, with the platelet concentration being higher than the concentration in the original blood collected. Dohan Ehrenfest $e t a l^{6} 7$ introduced the notion of leucocyte-rich PRP (LR-PRP) characterised by a leucocyte concentration higher than the whole blood baseline leucocyte level, whereas leucocyte-poor PRP (LP-PRP) or Pure PRP includes a leucocyte concentration lower than in whole blood. Accordingly, the platelet increase factor, corresponding to the platelet concentration increase in PRP compared with whole blood, is the most frequently described parameter in both scientific publications and manufacturer's promotional literature, and is thought to primarily influence the PRP efficacy. A platelet concentration in PRP below whole blood baseline level may not provide sufficient cellular response ${ }^{8}$ and platelet concentrations higher than six-fold compared with platelet whole blood baseline level may have an inhibitory effect on healing. ${ }^{9}$

Historical definitions from Marx and Dohan associated with the described 
influence of platelet concentrations in PRP efficacy have given rise to PRP classification ${ }^{10}{ }^{11}$ systems, but none of these classifications have been widely adopted.

In fact, the platelet increase factor in PRP compared with whole blood is directly linked to the volume of PRP obtained; these two factors should not be interpreted alone. We previously introduced the notion of platelet doses corresponding to the quantity of platelets and growth factors (GFs) hypothetically delivered at the injection site, as we previously described a positive correlation between platelet dose and quantity of GF. ${ }^{12}$ Based on the field of haematology, which first used cells as a therapy, cell doses are the most relevant parameter to assess clinical efficacy, and cell-dose effects are now clearly established. ${ }^{13}$ Otherwise, the current classifications of PRP do not take into account the red blood cell (RBC) content in PRP, which could represent a source of released reactive oxygen species that could also be clinically detrimental. That is why the global composition of PRP in platelets, leucocytes and RBCs, should be documented to analyse the clinical impact. Finally, to compare the efficiency of the PRP preparation device, the platelet recovery rate could be provided, allowing assessment of the platelet loss due to the process, although this parameter is not directly linked to clinical efficacy.

The purpose of this article is to introduce a standardised classification based on biological parameters classically used in the Cell Therapy field. This classification has been retrospectively applied to four publications comparing and describing biological characteristics of PRP devices available in Europe.

\section{Definition of PRP characterisation criteria and analysis of reported PRP preparations}

With the previous information being taken into consideration, the DEPA classification of PRP is based on four different components: (1) the Dose of injected platelets, (2) the Efficiency of the production, (3) the Purity of the PRP obtained, (4) the Activation process. The calculation of these parameters is only possible if complete cell counts are performed for both whole blood and PRP associated with the data of collected blood volume and injected PRP. We previously described the associated formulas. ${ }^{12}$

Through a retrospective analysis of four publications providing the mentioned data, we were able to classify 20 different PRP preparations using these variables. ${ }^{12}{ }^{14-16}$ Table 1 reports the protocol of PRP preparation from these publications.

\section{Dose of injected platelets}

The first part of the classification identifies the dose of injected platelets, which is calculated by multiplying the platelet concentration in PRP by the obtained volume of PRP. The injected dose of platelets should be measured in billions or millions of platelets and categorised as follows: A, very high dose of injected platelets of $>5$ billion; B, high dose of injected platelets, from 3 to 5 billion; C, medium dose of injected platelets, from 1 to 3 billion and, D, low dose of injected platelets, <1 billion.

Given the information available in the four publications, we were able to calculate the injected dose of platelets normalised with a baseline concentration of platelets at $200 \times 10^{9} / \mathrm{L}$. The production of PRP using a Selphyl device, described in the Kushida et $a l^{16}$ study, furnished 0.21 billion injected platelets, whereas the Magellan device characterised in the same study furnished 5.43 billion injected platelets, corresponding to a 25-fold increase. The complete data are provided in table 2.

\section{Efficiency of production}

The second criterion of classification corresponds to the efficiency of the production used to obtain PRP. The recovery rate in platelets (also called platelet capture efficiency) corresponds to the percentage of platelets recovered in the PRP from the blood. It is categorised as follows: A, high device efficiency if recovery rate in platelets is $>90 \%$; $\mathrm{B}$, medium device efficiency if recovery rate in platelets is from $70 \%$ to $90 \%$; C, low device efficiency if the recovery rate is from $30 \%$ to $70 \%$ and, $\mathrm{D}$, poor device efficiency for a recovery rate $<30 \%$. The retrospective application of this parameter to published data revealed that none of the processes described were of high efficiency. The recovery rates in platelets varied from $13.1 \%$ (the Selphyl device in the Kushida $e t a l^{16}$ study) to $79.3 \%$ (RegenLab in the Kaux et $a l^{15}$ study). The complete data are provided in table 2.

\section{Purity of the PRP}

The third criterion of the classification corresponds to the relative composition of platelets, leucocytes and RBCs in the obtained PRP. It presents the advantage of assessing the global purity of the PRP. It is categorised as follows: A, very pure PRP if percentage of platelets in the PRP compared with RBC and leucocytes is $>90 \%$; B, pure PRP if percentage of platelets in the PRP compared with RBC and leucocytes is from $70 \%$ to $90 \%$; C, heterogeneous PRP if percentage of platelets in the PRP compared with RBC and leucocytes is from $30 \%$ to $70 \%$; $\mathrm{D}$, whole blood PRP if percentage of platelets in the PRP compared with RBC and leucocytes is $<30 \%$. According to this criterion, the GPS II device furnishes a product highly contaminated by RBC with only $6 \%$ of platelets, which corresponds more or less to blood composition. Conversely, Curasan and Regen devices and the homemade preparation described by Kaux et $a l^{15}$ as well as the Selphyl device described by Kushida et al, give rise to very pure PRP.

It should be noted that leucocytes were at most only $1.64 \%$ (GPS II) in the final composition of the obtained PRP, but, the presence or absence of neutrophils is hotly debated and could be precised.

The complete data are furnished in table 2. 
Table 1 Protocol, volume collected and volume obtained from each preparation system provided in publications ${ }^{12}{ }^{14-16}$

\begin{tabular}{|c|c|c|c|c|c|}
\hline Reference & Device & $\begin{array}{l}\text { Number of } \\
\text { centrifugation } \\
\text { steps }\end{array}$ & Speed and time & $\begin{array}{l}\text { Collected } \\
\text { volume } \\
\text { of blood }(\mathrm{mL})\end{array}$ & $\begin{array}{l}\text { Volume of PRP } \\
\text { obtained }(\mathrm{mL})\end{array}$ \\
\hline \multirow[t]{5}{*}{ Kaux et $a l^{15}$} & Homemade & 1 & $180 \mathrm{~g} 10 \mathrm{~min}$ & 8 & 2.08 \\
\hline & Curasan & 2 & $\begin{array}{l}1000 \mathrm{~g} 10 \mathrm{~min}, \\
2300 \mathrm{~g} 15 \mathrm{~min}\end{array}$ & 8.5 & 1 \\
\hline & Plateltex & 2 & $\begin{array}{l}180 \mathrm{~g} 10 \mathrm{~min}, 1000 \mathrm{~g} \\
10 \mathrm{~min}\end{array}$ & 6 & 0.34 \\
\hline & GPS II & 1 & $180 \mathrm{~g} 15 \mathrm{~min}$ & 50 & 6.01 \\
\hline & RegenLab & 1 & $300 \mathrm{~g} 5 \mathrm{~min}$ & 6 & 3.068 \\
\hline \multirow[t]{3}{*}{ Castillo et al ${ }^{14}$} & Cascade & 1 & $1100 \mathrm{~g} 6 \mathrm{~min}$ & 18 & 7.5 \\
\hline & GPS III & 1 & $1100 \mathrm{~g} 15 \mathrm{~min}$ & 55 & 6 \\
\hline & Magellan & 1 & $1200 \mathrm{~g} 17 \mathrm{~min}$ & 26 & 6 \\
\hline \multirow{5}{*}{ Magalon et al ${ }^{12}$} & Selphyl & 1 & $1100 \mathrm{~g} 6 \mathrm{~min}$ & 8 & 4.1 \\
\hline & RegenPRP & 1 & $1500 \mathrm{~g} 9 \mathrm{~min}$ & 8 & 3.1 \\
\hline & Mini GPS III & 1 & $3200 \mathrm{rpm} 15 \mathrm{~min}$ & 27 & 3.21 \\
\hline & Arthrex & 1 & $1500 \mathrm{rpm} 5 \mathrm{~min}$ & 11 & 4.03 \\
\hline & Homemade & 2 & $\begin{array}{l}130 \mathrm{~g} 15 \mathrm{~min}, 250 \mathrm{~g} \\
15 \mathrm{~min}\end{array}$ & 30 & 3.41 \\
\hline \multirow[t]{7}{*}{ Kushida et $a l^{16}$} & JP200 & 2 & $\begin{array}{l}1000 \mathrm{~g} 6 \mathrm{~min}, 800 \mathrm{~g} \\
8 \mathrm{~min}\end{array}$ & 20 & 1 \\
\hline & GLO & 2 & $\begin{array}{l}1800 \mathrm{~g} 3 \mathrm{~min}, 1800 \mathrm{~g} \\
6 \mathrm{~min}\end{array}$ & 8.5 & 0.6 \\
\hline & Magellan & 2 & $\begin{array}{l}610 \mathrm{~g} 4 \mathrm{~min}, 1240 \mathrm{~g} \\
6 \mathrm{~min}\end{array}$ & 60 & 3 \\
\hline & Kyocera & 2 & $\begin{array}{l}600 \mathrm{~g} 7 \mathrm{~min}, 2000 \mathrm{~g} \\
5 \mathrm{~min}\end{array}$ & 20 & 2 \\
\hline & Selphyl & 1 & $525 \mathrm{~g} 15 \mathrm{~min}$ & 8 & 2 \\
\hline & MyCells & 1 & $2054 \mathrm{~g} 7 \mathrm{~min}$ & 10 & 1 \\
\hline & Dr. Shin & 1 & $1720 \mathrm{~g} 8 \mathrm{~min}$ & 8.5 & 1 \\
\hline
\end{tabular}

\section{Activation process}

Finally, addition of exogenous clotting factor to activate platelets is already described in available classifications $^{10}{ }^{11}$ and should be mentioned. Addition of calcium chloride allows the release of GFs in a liquid form and PRP gel can be obtained by mixing PRP with autologous thrombin and calcium chloride. As this activation depends on the treatment indications and physician's decision, we did not compare it in this analysis.

\section{DISCUSSION}

Several authors have demonstrated substantial differences in the content of platelet concentrates produced by various automated and manual protocols described in the literature. ${ }^{12}{ }^{14-16}$ To face this issue, classifications recently appeared and are focused on two parameters: the increased platelet and leucocyte factor compared with whole blood. This presents some drawbacks: (1) the volume is not taken into account, directly influencing the concentration. As an example, Plateltex, described by Kaux et al, delivered an increased platelet factor of only 3.43 , because a very small final volume of $0.34 \mathrm{~mL}$ was obtained. The corresponded dose injected was only 0.23 billion. (2) They do not assess the efficacy of the process allowing the comparison of one preparation with another and (3) they do not take into account PRP as a global product containing not only platelets and leucocytes, but also RBCs. The major challenge of PRP preparation is to remove RBCs and reverse the initial composition of blood (95\% of RBCs), and this is sometimes not achieved at all-an example is the GPS II device, globally composed of $93.9 \%$ RBCs.

Through the introduction of new parameters (dose of injected platelets, recovery rate in platelets and the relative composition of PRP), the DEPA classification circumvents these issues. Thus, a PRP preparation reaching an 'AAA' DEPA score will mean that a very high dose of platelets was injected ( $>5$ billion) with little contamination from RBCs, and that the preparation was optimal with minor loss of platelets from blood. A limitation to this 'ABCD' scoring system is that an A score will often be evaluated as better than a B, C or D score, whereas the impact of platelet dose and purity remains unknown.

It should be noted that devices corresponding to a very high dose of injected platelets will necessarily correspond to an important collected volume (minimum $30 \mathrm{~mL}$ ). It will be also be difficult to reach a high dose of platelets for indications necessitating very small 
Table 2 Application of DEPA score to 20 PRP preparations in which biological characteristics are available on publications indexed in PubMed

\section{DEPA classification}

Dose of injected platelets (billions)

Kaux etali

A $>5$

Kaux et $a l^{15}$

Homemade
Curasan

Curasan

Plateltex

GPS II

Castillo et $a l^{14}$

RegenLab

Cascade

GPS III

Magellan

Magalon et al ${ }^{12}$

Selphyl

RegenPRP

Mini GPS III

Arthrex

Homemade

Kushida et al $^{14} \quad$ JP200

GLO

Magellan

Selphyl

MyCells

Dr. Shin

\begin{tabular}{lll}
\hline A & $>5$ & Very high dose \\
B & $3-5$ & High dose
\end{tabular}

$\begin{array}{lll}\text { C } & 3-5 & \text { High dose } \\ \text { C } & 1-3 & \text { Medium dose }\end{array}$

D $<1 \quad$ Low dose

D 0.74

Low dose

0.55

0.23

Low dose

Low dose

Medium dose

Low dose

Medium dose

Medium dose

High dose

Low dose

Low dose

Medium dose

Medium dose

Medium dose

Medium dose

Low dose

Very high dose

High dose

Low dose

Low dose

Low dose

Efficiency of the process
(platelet recovery rate \%)

composition in platelets \%)

$\begin{array}{llllll} & >90 & \text { High } & & >90 & \text { Very pure PRP } \\ \text { B } & \mathbf{7 0 - 9 0} & \text { Medium } & \text { B } & \mathbf{7 0 - 9 0} & \text { Pure PRP }\end{array}$

B 70-90 Medium

C 30-70 Low

D $\quad<30 \quad$ Poor

C $46.2 \quad$ Low

Low

19.4

22.8
79.3

67.5

67.5
22.6

65.8

59.5

61.7

34.6

48.0

30.2

26.0

37.4

45.3

78.1

13.1

48.8

45.9

C

Poor

Poor

Medium

Low

Poor

Low

Low

Low

Low

Low

Low

Poor

Low

Low

Medium

Poor

Low

Low

Pure PRP Whole blood PRP

A $\quad 97.7 \quad$ Very pure PRP

Pure PRP

Whole blood PRP

87.5
6.0

97.5

81.5

27.0

60.4

73.9

46.0

51.8

81.0

80.7

19.6

38.2

38.2
32.9

29.4

99.7

87.3

18.8

Very pure PRP

Pure PRP

Whole blood PRP

Pure PRP

Heterogeneous PRP

Pure PRP

Pure PRP

Whole blood PRP

Heterogeneous PRP

Whole blood PRP

Very pure PRP

Pure PRP

Whole blood PRP

D $\quad 0.78$

DEPA, Dose of injected platelets, Efficiency of production, Purity of the PRP, Activation of the PRP; PRP, platelet-rich plasma.

Heterogeneous PRP Final DEPA score

A $90.3 \quad$ Very pure PRP DCA

DCA

DDB

Heterogeneous PRP

Heterogeneous PRP

Heterogeneous PRP

CDD

DBA

CCB

CDD

$B C C$

DCB

DCC

$\mathrm{CCC}$

$\mathrm{CCB}$

CCB

CDD

DCC

ACC

BBD

DDA

DCB

DCD 
volume (ie, intratendinous requirements) and could represent a challenge for future development to manufacturers of PRP production devices.

The clinical relevance of the DEPA classification remains to be evaluated in clinical studies and review of clinical trials. This point is still limited by the absence of characterisation in the majority of clinical trials. A few randomised clinical trials ${ }^{17} 18$ performed a characterisation of the injected PRP, but these were restricted to the publication of platelet concentration in PRP, and did not broach the subject of the clinical impact of RBCs and leucocytes in PRP. Future clinical studies should describe the reported volumes, dose of platelets as well as the overall composition of whole blood and PRP, and the number of applications of PRP, in which the DEPA classification could be considered as a tool (1) to determine the clinical impact of the huge variability of PRP composition and (2) to assess the quality of PRP production.

Contributors JM, AC and BB performed the review. ML, AS and LG assisted with the review and edited the manuscript. JV and FS edited the final manuscript.

Competing interests None declared.

Provenance and peer review Not commissioned; externally peer reviewed.

Open Access This is an Open Access article distributed in accordance with the Creative Commons Attribution Non Commercial (CC BY-NC 4.0) license, which permits others to distribute, remix, adapt, build upon this work noncommercially, and license their derivative works on different terms, provided the original work is properly cited and the use is non-commercial. See: http:// creativecommons.org/licenses/by-nc/4.0/

\section{REFERENCES}

1. Sanchez M, Anitua E, Azofra J, et al. Intra-articular injection of an autologous preparation rich in growth factors for the treatment of knee OA: a retrospective cohort study. Clin Exp Rheumatol 2008;26:910-13.

2. Radice F, Yánez R, Gutiérrez V, et al. Comparison of magnetic resonance imaging findings in anterior cruciate ligament grafts with and without autologous platelet-derived growth factors. Arthroscopy 2010;26:50-7.
3. de Vos RJ, Weir A, van Schie HT, et al. Platelet-rich plasma injection for chronic Achilles tendinopathy: a randomized controlled trial. JAMA 2010;303:144-9.

4. Reurink G, Goudswaard GJ, Moen MH, et al. Platelet-rich plasma injections in acute muscle injury. N Engl J Med 2014;370:2546-7.

5. Marx RE. Platelet-rich plasma (PRP): what is PRP and what is not PRP? Implant Dent 2001:10:225-8.

6. Dohan Ehrenfest DM, Rasmusson L, Albrektsson T. Classification of platelet concentrates: from pure platelet-rich plasma (P-PRP) to leucocyte- and platelet-rich fibrin (L-PRF). Trends Biotechnol 2009;27:158-67.

7. Dohan Ehrenfest DM, Bielecki T, Del Corso M, et al. Shedding light in the controversial terminology for platelet-rich products: platelet-rich plasma (PRP), platelet-rich fibrin (PRF), platelet-leukocyte gel (PLG) preparation rich in growth factors (PRGF), classification and commercialism. J Biomed Mater Res A 2010;95:1280-2.

8. Haynesworth S, Kadiyala S, Liang L. Mitogenic stimulation of human mesenchymal stem cells by platelet release suggest a mechanism for enhancement of bone repair by platelet concentrates. Boston, MA: 48th Meeting of the Orthopedic Research Society, 2002.

9. Weibrich G, Hansen T, Kleis W, et al. Effect of platelet concentration in platelet-rich plasma on peri-implant bone regeneration. Bone 2004;34:665-71.

10. DeLong JM, Russell RP, Mazzocca AD. Platelet-rich plasma: the PAW classification system. Arthroscopy 2012;28:998-1009.

11. Mishra A, Harmon K, Woodall J, et al. Sports medicine applications of platelet rich plasma. Curr Pharm Biotechnol 2012;13:1185-95.

12. Magalon J, Bausset $\mathrm{O}$, Serratrice $\mathrm{N}$, et al. Characterization and comparison of 5 platelet-rich plasma preparations in a single donor model. Arthroscopy 2014;30:629-38.

13. Gluckman E, Rocha V, Boyer-Chammard A, et al. Outcome of cord-blood transplantation from related and unrelated donors. Eurocord Transplant Group and the European Blood and Marrow Transplantation Group. N Engl J Med 1997; 337:373-81.

14. Castillo TN, Pouliot MA, Kim HJ, et al. Comparison of growth factor and platelet concentration from commercial platelet-rich plasma separation systems. Am J Sports Med 2011;39:266-71.

15. Kaux JF, Le Goff C, Seidel L, et al. [Comparative study of five techniques of preparation of platelet-rich plasma]. Pathol Biol (Paris) 2011:59:157-60.

16. Kushida S, Kakudo N, Morimoto N, et al. Platelet and growth factor concentrations in activated platelet-rich plasma: a comparison of seven commercial separation systems. J Artif Organs 2014;17:186-92.

17. Patel S, Dhillon MS, Aggarwal S, et al. Treatment with platelet-rich plasma is more effective than placebo for knee osteoarthritis: a prospective, double-blind, randomized trial. Am J Sports Med 2013:41:356-64.

18. Rayegani SM, Raeissadat SA, Taheri MS, et al. Does intra articular platelet rich plasma injection improve function, pain and quality of life in patients with osteoarthritis of the knee? A randomized clinical trial. Orthop Rev (Pavia) 2014;6:5405. 